\title{
The African swine fever modelling challenge: objectives, model description and synthetic data generation
}

Sébastien Picault ${ }^{*}$, Timothée Vergne ${ }^{2}$, Matthieu Mancini ${ }^{1,2}$, Servane Bareille ${ }^{1,2}$, Pauline Ezanno $^{1}$

${ }^{1}$ INRAE, Oniris, BIOEPAR, 44300, Nantes, France

\author{
${ }^{2}$ INRAE, ENVT, IHAP, Toulouse, France
}

${ }^{*}$ Corresponding author - BIOEPAR, Oniris, La Chantrerie, route de Gachet, CS 40706, 44307

Nantes cedex 3, France

Emails: sebastien.picault@inrae.fr, timothee.vergne@envt.fr, matthieu.mancini@inserm.fr, servanebareille@,hotmail.fr, pauline.ezanno@inrae.fr

\begin{abstract}
African swine fever (ASF) is an emerging disease currently spreading at the interface between wild boar and pig farms in Europe and Asia. Current disease control regulations, which involve massive culling with significant economic and animal welfare costs, need to be improved. Modelling enables relevant control measures to be explored, but conducting the exercise during an epidemic is extremely difficult. Modelling challenges enhance modellers' ability to provide timely advice to policy makers, improve their readiness when facing emerging threats, and promote international collaborations. The ASF-Challenge, which ran between August 2020 and January 2021, was the first modelling challenge in animal health. In this paper, we describe the objectives and rules of the challenge. We then demonstrate the mechanistic multi-host model that was used to mimic as accurately as possible an ASF-like epidemic, provide a detailed explanation of the surveillance and intervention strategies that generated the synthetic data, and describe the different management strategies that were assessed by the competing modelling teams. We then outline the different technical steps of the challenge as well as its environment. Finally, we synthesize the lessons we learnt along the way to guide future modelling challenges in animal health.
\end{abstract}

Keywords: epidemiology; control measures; agent-based model; spatial model; swine-wild boar contacts

\section{Highlights}

- The $1^{\text {st }}$ modelling challenge in animal health mimics ASF spread with synthetic data

- A mechanistic spatially explicit stochastic model was developed to represent ASF spread and control

- Synthetic data concerned date and location of detected cases in pigs and wild boar

- The wildlife-livestock interface is crucial in infectious animal disease spread 


\section{Introduction}

To raise livestock in a manner that is sustainable and respects animal welfare, animal health must be managed, including infectious diseases which spread extensively between animal populations (Ezanno et al. 2020). Livestock diseases are currently being affected by changes at both the farm and global level. Societal demand for more outdoor livestock farming may increase contacts between livestock and wildlife, and thus pathogen spread at this interface (Gortázar et al. 2007). Movements of persons, animals and animal products also are intensifying, and together with climate changes, are favouring the emergence and spread of new pathogens (Parham et al., 2015; Saker et al., 2004). These developments are impacting animal health on a massive scale, and may affect public health when zoonoses are concerned.

African swine fever (ASF) is a good example of an emerging animal disease spreading at the interface between wildlife and livestock (Sánchez-Cordón et al., 2019; EFSA, 2021). This nonzoonotic viral disease originates from sub-Saharan Africa, where it is asymptomatically transmitted among warthogs and bushpigs. Wild boar and domestic pigs are susceptible hosts, with mortality rates of almost $100 \%$ for virulent strains such as the one currently circulating on the Eurasian continent (Dixon et al., 2020). Due to this high mortality rate, ASF has a tremendous impact on swine production, on the economy of livestock systems and on international trade, and neither a vaccine nor a treatment is available (Dixon et al., 2020). Therefore, since 21 April 2021, ASF has been categorized as an A+D+E disease under EU legislation. This means that immediate eradication measures must be taken as soon as the disease is detected on a territory (A), preventive actions are needed to prevent the disease on account of its entry in the EU (D), and surveillance protocols are mandatory within the EU (E). In 2007, a highly virulent strain was introduced into Georgia (Rowlands et al. 2008) and then spread to the Russian Federation, from where it disseminated towards Europe and Asia (Dixon et al., 2020). It is now considered endemic in several countries of Eastern Europe and East and Southeast Asia. As the virus can spread internationally via geographical proximity or due to the movements of persons, swine and swine products, ASF has become one of the most important livestock infectious disease threat for most countries (Vergne et al., 2017).

Every health crisis, e.g., the recent pandemic of COVID-19 in humans (Holmdahl and Buckee, 2020), the foot-and-mouth disease epidemics in livestock in the UK (Keeling, 2005) and the ash dieback epidemics in European ash trees (Coker et al., 2019), highlights the need for robust epidemiological knowledge and predictive tools to better cope with health uncertainty. Developing models that forecast disease spread is key to better understanding epidemics (Grassly \& Fraser, 2008). Models are not new in epidemiology (Hamer, 1906), but with the computer revolution, and in the age of big data where information is shared almost in real time, this field has been revolutionized (Rosenfeld et al., 2013).

In the case of ASF, simulation models have been developed to assess stricter regulations in Europe (Halasa et al., 2016). Their pessimistic results promoted the use of alternative measures (Lange, 2015), such as barriers and over-hunting in retention zones used in Belgium (EFSA, 2020). However, deciding which measures to implement remains challenging, especially once an epidemic has started and decisions need to be taken promptly. To enhance global preparedness for ASF epidemics, the livestock/wildlife interface first must be explicitly considered, and a capacity to assess different and combined control measures must then be developed (Hayes et al., 2021).

A wide variety of models can be considered to represent an epidemiological situation (Chretien et al., 2014; Nsoesie et al., 2014; Holmdahl and Buckee, 2020). They are generally categorized as phenomenological models (e.g., time series, generalized linear models, survival models, etc.) 
and mechanistic models (e.g., SIR-like models, multi-agent systems, etc.). While the advantages and disadvantages of each model type are generally known, it is difficult to decide a priori whether one type is systematically more suitable than another for a given situation. Comparing models is therefore essential, but the task is difficult. Models may not use the same data or cover the same periods of the epidemic. The geographical areas over which predictions are made also can differ. These limitations appeared in the comparison of models used during the Ebola epidemics in West Africa (Chretien et al., 2015), and during influenza epidemics (Chretien et al., 2014; Nsoesie et al., 2014). In addition, a lack of cooperation between modellers and public health decision-makers, particularly due to communication problems, is often deplored (Metcalf et al., 2015). Modellers also report difficulties in modelling the spread of a disease in real time, especially in the early stages (Van Kerkhove \& Ferguson, 2012). More preparation is clearly needed to face these challenges (Johansson et al., 2019), better compare the predictive effectiveness of different models, advance the field of epidemiological modelling, and assist public health decision making.

To improve the accuracy of model predictions and cooperation between actors, the scientific community has developed relatively short competitions known as modelling challenges. The first challenge, organized in 1994, was on protein structure prediction (Friedberg et al., 2015). In epidemiological modelling, the first challenge was organized in 2013 on seasonal influenza in the US, and has since been annually renewed (Reich et al., 2019; Viboud and Vespignani, 2019). Three other challenges also were organized in 2015 on Ebola (Viboud et al., 2018), Chikungunya (Del Valle et al., 2018), and Dengue (Johansson et al., 2019). Thus far, however, no challenge has involved an animal disease. Modelling the transmission of such infectious diseases needs to address specific features, including the spatial distribution of farms and the difficulty of monitoring infectious diseases in wildlife.

This paper aims to introduce the ASF modelling challenge (ASF-Challenge). It presents the objective of the challenge, the stochastic, agent-based and spatialized epidemiological model developed to represent ASF spread at the interface between domestic pigs and wild boar, and the synthetic data produced using this model to mimic an ASF epidemic for the ASF-Challenge. The performance of the different models participating in the ASF-Challenge are presented in the last article from this special issue (Ezanno et al. submitted).

\section{Methods}

\subsection{ASF-Challenge characteristics}

The objective of this first modelling challenge in animal health was to create a unique and stimulating environment to enhance the ability of modellers to advise policy makers in a timely manner. More specifically, it aimed at improving the readiness of modelling teams when facing emerging threats such as ASF and promoting international collaborations.

The preparation for the ASF-Challenge started in July 2019. This preparation phase was used to define the ASF-Challenge objectives, build the "mother model" that generated the synthetic data used in the challenge, and clarify what was expected from the participating teams. The ASF-Challenge itself was initially planned to take place between March and July 2020, but it was postponed due to the COVID-19 pandemic. It was finally launched on 27 August 2020 and lasted until 13 January 2021. Once the ASF-Challenge itself had ended, a series of internal workshops were organized to obtain feedback from the challenge teams and present the different models that were used. This also provided an opportunity to synthesize the challenge 
outputs and reflect on the lessons learnt. These are presented in the last article from this special issue (Ezanno et al. submitted).

The data that were provided to the challenge participants were generated by a detailed agentbased model that was fed population data (spatial distribution of the host populations, movements of live pigs, etc.) and parametrized with various key parameters defining transmission processes as well as intervention strategies. This will be described in detail in subsequent paragraphs. To be useful for the ASF-Challenge, we aimed to generate ASF-like epidemic trajectories with the following characteristics: first outbreak detected in a domestic pig herd at the vicinity of a forest area and less than 200 days after the first infection, more than 250 infected wild boar at the time of the first detection, outbreaks reported in both domestic pig farms and wild boar over the course of the epidemic, apparent epidemic duration of more than 100 days, progressive diffusion towards a forest area with a high density of wild boar and an apparent successful control of the disease (i.e., less than 500 infected wild boar outside the fence when implemented, more than 250 infected wild boar 110 days after the first detection but less than 30 infected wild boar 230 days after first detection) at the end of the ASFChallenge.

To ensure that the model represented a realistic setting of hosts and could reproduce an ASFlike epidemic with sufficient accuracy, we relied on a small group of French experts with knowledge on pig production, wild boar ecology and African swine fever epidemiology. These experts suggested relevant data sources and made suggestions regarding the assumptions of the model (population distributions, movement data, transmission processes, etc.).

\subsection{Data used to feed the model}

The simulated epidemic occurred on a hypothetical island that was created by merging two French administrative regions, naming Auvergne-Rhone-Alpes and Occitanie. All polygons and land use data were obtained from the DIVA-GIS website (https://www.diva-gis.org/) and all land use types were aggregated into three types: agricultural, forest and urban areas. The model used as inputs the spatial locations of individual wild boar and domestic pig farms which were simulated based on land use data.

To simulate wild boar distribution, we obtained the hunting bags at the department level from the Office Français de la Biodiversité, and assumed that a hunting season would reduce the wild boar population by half, so that the total wild boar population size was twice the hunting bag size at the level of the whole island. The location of individual wild boars was then randomly simulated for each of the 500,366 wild boar, assuming that $18 \%, 80 \%$ and $2 \%$ of wild boar would be in agricultural, forest and urban areas, respectively. Their geographical coordinates were assumed to represent the centre of their home range. The spatial distribution of individual wild boar was then summarized as department-level hunting bags, assuming again that hunting bag sizes represented only half the size of the wild boar population in each department.

To simulate a regionalization and a spatially heterogeneous distribution of the 4,775 pig farms, we assumed that 1) $33 \%$ of farms would be in the Auvergne-Rhone-Alpes region while the remaining $67 \%$ would be in the Occitanie region, and 2) $85 \%, 10 \%$ and $5 \%$ of pig farms would be in agricultural, forest and urban areas, respectively. Based on these specificities, we simulated geographical coordinates for the 4,775 pig farms that were registered in the two French regions. Different farm characteristics, including whether they were commercial or backyard, breeder, finisher or breeder-finisher, whether the pigs had access to an outdoor area, 
farm capacity and the regrouping of different farms into the same pig company were also simulated successively. All of this information was used to generate a biosecurity score which was used to vary a farm's susceptibility to, and transmissibility of, ASF (see below). Live-pig movement data between farms also were simulated over time, assuming that breeder and breeder-finisher farms could send pigs to finisher or breeder-finisher farms, that a breederfinisher farm was more likely to fatten its own pigs than to send them to another farm, that farms belonging to a pig company were more likely to send pigs to farms from the same company than to farms not belonging to the same company, and that the total number of pigs in a farm at a given time could not exceed the size of the farm.

\subsection{Model used to produce synthetic epidemiological data}

To produce these synthetic data, we needed a very detailed model that was expected to be more complex than any model used by the ASF-Challenge teams. Hence, we designed a stochastic mechanistic model which integrated the most up-to-date fine-grained knowledge and assumptions with regard to ASF spread and control in wild boar and domestic pigs. This model was in discrete time, with a time step of one day, spatially explicit and agent-based with three types of agents: the pig herd (as a compartment-based sub-model), the individual wild boar with its location, and the whole hypothetical island as a metapopulation. The model was implemented using a recent modelling approach, EMULSION (Picault et al., 2019), which helps to make model components explicit and to reduce the amount of code required.

\subsubsection{Population dynamics and infection spread}

The dynamics of the domestic pig population was determined only by the trade movements between farms depending on their type (breeders, finishers and breeder-finishers), neglecting natural mortality and replacing sold animals with the same number of new ones (representing births in breeders or breeder-finishers, and purchases in finishers or breeder-finishers), without considering the detailed herd structure (e.g., batch management). In wild boar, we considered natural mortality and hunting through constant rates. We considered no birth during the simulated period, as the epidemic was assumed to occur during the hunting season, i.e., after the reproductive period (Vetter et al 2020). The hunting rate was calculated to remove $50 \%$ of the wild boar population during the hunting season (in 8 months), consistent with how the hunting bags were used to generate the wild boar distribution (Jori et al., 2021). All of the parameters involved in the dynamics of pig farms and the wild boar population are provided in the supplementary information (SI5).

All epidemiological units (pig herds and individual wild boar) were subject to the same infectious process, with the following states for animals in the units: susceptible (S), exposed (E) where animals started being infectious but were still asymptomatic, fully infectious and symptomatic (I). All infected animals eventually died, producing an infectious carcass (C). We also assumed that wild boar were subject to natural mortality, giving either a healthy (D) or infectious (C) carcass depending on their health state at death. The durations in states E, I, C, $\mathrm{D}$ were distributed exponentially. In pig herds, carcasses were removed the next day, whereas infectious (C) or healthy (D) wild boar carcasses could remain in the environment for several weeks or months until they naturally decomposed or were removed when found by a passer-by. Within pig herds, we assumed a frequency-dependent force of infection, exposed individuals contributing to half the level of infectious animals or infectious carcasses. We also assumed a higher transmission rate in backyard farms than in commercial farms. All of the parameters involved in the infection process are provided in supplementary information (SI5).

For pig farms, we considered the following transmission pathways between epidemiological units: 1) arrival of an infected pig from an infected farm in another farm, through trade 
movements, 2) contact with an infectious wild boar and 3) indirect contact with an infectious farm due to visits, exchange of agricultural material, etc. For wild boar, we considered: 4) contact with an infectious live wild boar, 5) contact with an infectious wild boar carcass, 6) contact with an infectious pig farm. All of the transmission pathways assuming a contact with a farm were adjusted based on the biosecurity score of the farm.

Trade movements were pre-computed as a temporal directed graph between farms, accounting for realistic animal movements in typical European pig farming systems. All of the five other transmission pathways were spatially explicit, using an exponential transmission kernel function of the square of the distance between epidemiological units. This approach was also used to represent between-farm indirect transmission. For each of the five spatially explicit transmission pathways, we considered different values for the kernel parameter (Table S1). We also assumed that contacts between wild boar and pig farms could occur only for farms providing animals access to the outdoors. Finally, we assumed that the contribution of infected pig farms to other susceptible epidemiological units was a function of their within-farm prevalence and their biosecurity level. The resulting forces of infection experienced by each possible epidemiological unit from other infectious units are summarized in the supplementary information (Table S2).

\subsubsection{Intervention strategies}

The model represented several detection methods depending on the characteristics of each epidemiological unit. We assumed that all tests were perfectly sensitive and specific to focus on the interplay between intervention strategies, detection, and control. All of the parameters involved in the intervention strategies are provided in the supplementary information (SI3-5).

Prior to any primary case, detection relied only on passive surveillance. In wild boar, we assumed that each carcass could be found, reported and tested each day with a constant and low probability. In infected farms, each infected pig could be detected and tested each day while being in infectious state (I) with a constant probability, and at death. Detection probabilities varied depending on the farm type (higher in commercial farms, lower in backyard) and increased in all pig farms after the detection of the primary case to account for enhanced vigilance (Table S3).

The model incorporated all current European regulatory measures and triggered them as soon as a primary case was detected in the simulation. All animals in detected pig farms were culled, and the delay between detection and culling was fixed. A protection zone and a surveillance zone were established (Table S6), both subject to a trade ban and increased vigilance (increased detection probabilities and biosecurity scores). Farms which had exchanged animals with a detected farm during the previous three weeks (called "traced farms" in what follows) also were subjected to the same restrictions and vigilance as in the protection zone, with the same duration. Culled farms could be repopulated after a fixed period of time (Table S6).

Infected wild boar carcasses found in the environment were removed immediately and led to an active search for other infected carcasses (Table S6). During this active search, each wild boar carcass (either infected or disease-free) could be found with an increased probability compared to carcasses outside the search zone. New infected carcasses triggered themselves new active search operations (assuming no logistic constraints). After the detection of the primary case, it was also assumed that part of hunted wild boar were tested (Table S6).

In addition to regulatory measures, alternative interventions were triggered in the simulation (Table S7). First, as the forest near the primary case could be considered a main threat for virus diffusion, $300 \mathrm{~km}$ of fences were installed 60 days after the detection of the primary case. The 
hunting pressure was increased within the fenced area as well as in a buffer area outside the fences, aiming to remove $90 \%$ of wild boar by the end of the hunting period (instead of $50 \%$ in other areas). In all areas with increased hunting, all hunted animals were tested, the active search for wild boar carcasses was suspended, and the probability of finding wild boar carcasses by passive surveillance was much higher than before. The increased hunting effort in the buffer area occurred during two months. Second, 90 days after the detection of the primary case and until the end of the simulation, when an infected wild boar was found (either through hunting or as a carcass), all animals from nearby farms (Table S7) were culled preventively and tested (leading to the installation of protection and surveillance areas and trade contact tracing if positive).

\subsubsection{Stochastic simulations, model outputs, and selected synthetic data}

The virus was initially introduced shortly before the beginning of the hunting season (Table S4), through an exposed wild boar located near a forest close to the centre of the hypothetical island, all other epidemiological units being fully susceptible.

In addition to the numbers in each health state for each epidemiological unit, the model kept track of all events (infection; detection with date; cause of removal: active search, hunting, culling; test). These fine-grained model outputs were used to (i) analyze the stochastic trajectories and select a relevant one for the challenge, and (ii) reconstruct simulated epidemiological data as time series given to the ASF-Challenge participants. We calculated the maximal geographical extent of the spatial distribution of cumulative cases, defined as a rectangle whose corners were the most extreme coordinates of the infected wild boar (respectively pig farms) encountered during the game (increasing function). Finally, we assessed the probability of infection of each pixel $\left(5 \times 5 \mathrm{~km}^{2}\right.$ squares $)$, defined by the proportion of model repetitions where the pixel had at least one infected pig farm, live wild boar, or carcass.

To find trajectories with ASF-like dynamics that were realistic enough for the challenge, 500 stochastic repetitions were run. First, simulations without any detection (40/500) or with a date of primary case detection later than 200 days after virus introduction $(9 / 500)$ were discarded as unfit for the challenge. Among the 451 remaining simulations, five selection criteria were defined (SI6, Table S8). Seven replicates met all five selection criteria, among which the one actually selected for the ASF-Challenge was chosen at random.

\section{Results}

\subsection{General predictions of the model}

\subsubsection{Temporal dynamics}

The infection dynamics was highly variable among the 451 stochastic model repetitions, despite similar initial conditions. This was especially true in wild boar where the number of cases was huge (Fig. 1). It grew rapidly in most of the repetitions, within a large range both for the date and amplitude of the epidemic peak. Regulated measures enabled the spread to be limited and reach a plateau of cases before the fences were implemented. Fences and increased hunting were required to decrease the number of cases in wild boar and the associated exposure of pig herds. Finally, a rebound of the epidemics was highly probable after the increased hunting had stopped (while other control measures still were implemented).

The seven repetitions meeting all selection criteria (Tab. S8) showed small epidemics in pig herds and intermediate ones in wild boar (Fig. 2). The last criteria $(<30$ infected live wild boar 230 days after first detection) led to what was almost disease fade-out (Fig. 1). 


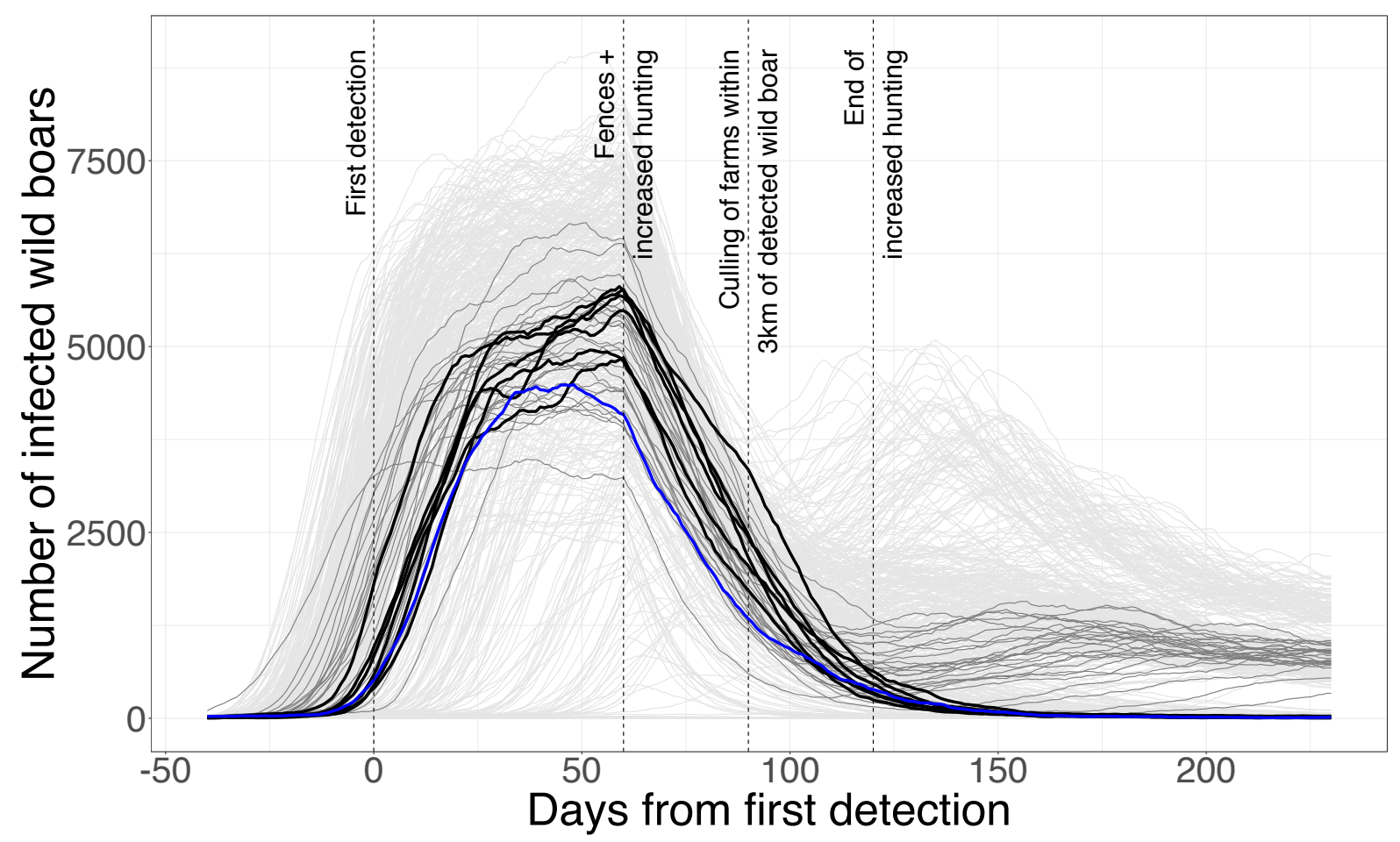

Figure 1. Temporal dynamics of the number of live infected wild boars (exposed + infectious) for 451 stochastic repetitions of the model. Blue: repetition selected for the ASFChallenge; black: the 6 other repetitions meeting the 5 selection criteria; dark grey: the 34 repetitions meeting all the criteria except the last one $(<30$ infected wild boars 230 days after first detection). Vertical dotted lines: changes in control interventions.

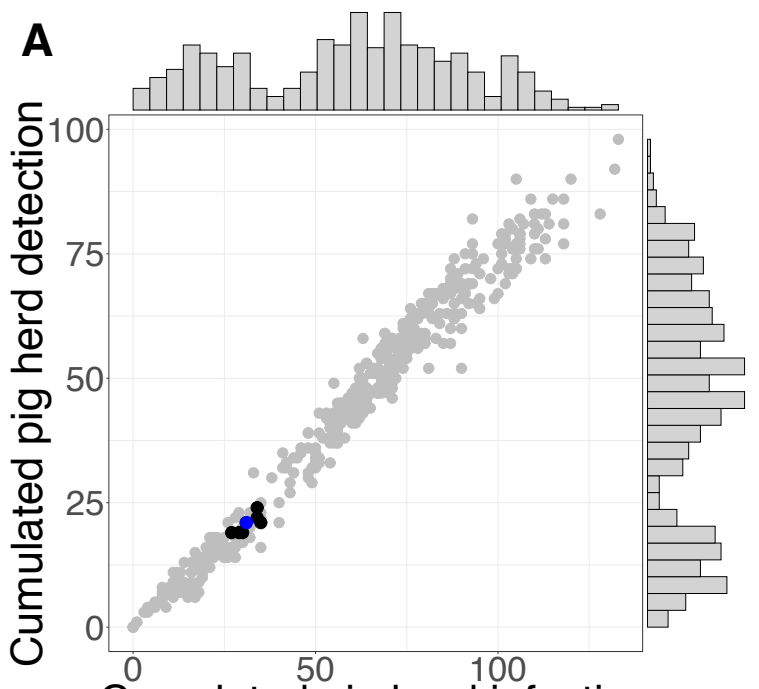

Cumulated pig herd infection

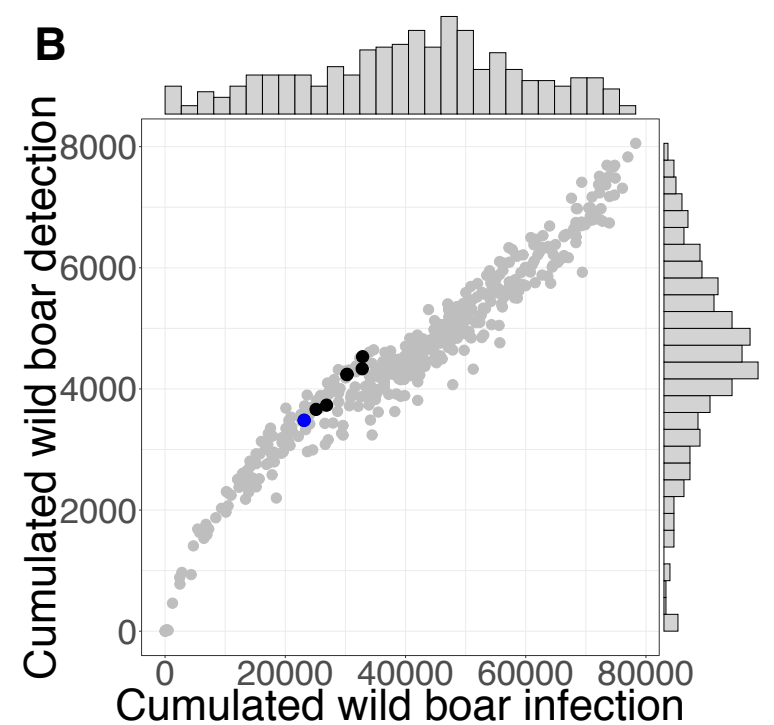

Figure 2. Distribution of the cumulative epidemic size and detection up to 230 days after the first detection for 451 stochastic repetitions of the model. A: pig herds; B: wild boar; blue: repetition selected for the ASF-Challenge; black: the 6 other repetitions meeting the five selection criteria; grey: other 444 repetitions. 


\subsubsection{Spatial dynamics}

The maximal geographical extent of the epidemics was also highly variable among repetitions, both in the wild boar and pig herd populations (Fig. 3). The seven repetitions satisfying all selection criteria showed a low spatial spread in the wild boar population (Fig. 3B), very close to the surface of the fenced area. In contrast, they showed a much more variable spatial spread in pig herds (Fig. 3A), in relation with commercial movements of infected animals before source herds were detected. These long-distance spreading events in pig herds did not impact the spatial spread of the disease in wild boar, highlighting a low exposure of wild boar to infectious pig herds.

A

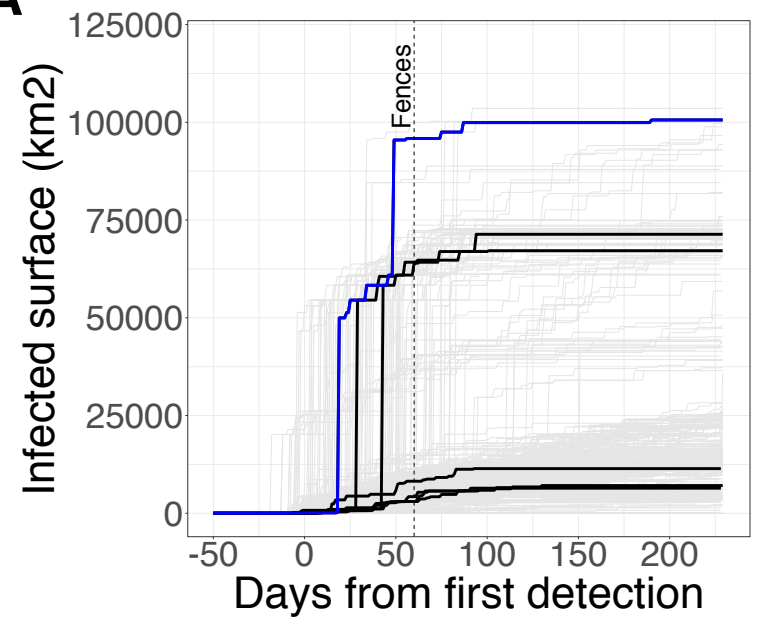

B

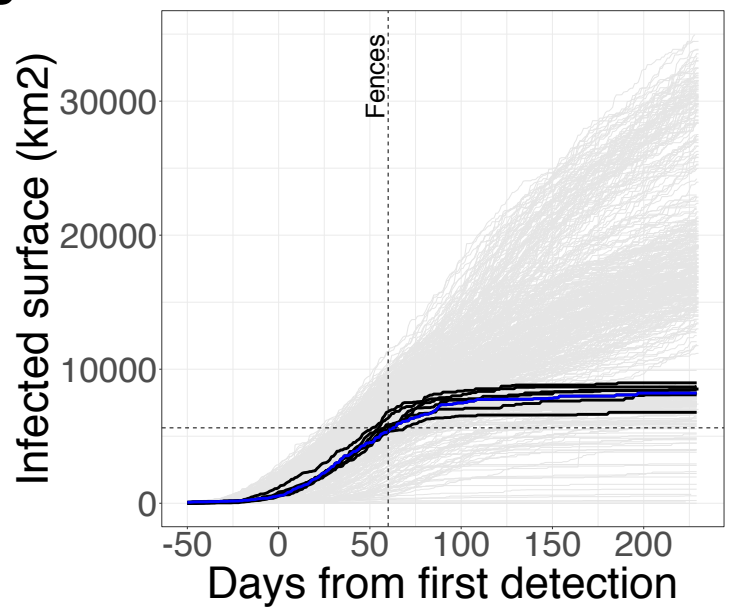

Figure 3. Temporal evolution of the maximal geographical extent of the spatial distribution of cumulative cases for 451 stochastic repetitions of the model. A: pig herds; B: wild boar; blue: repetition selected for the ASF-Challenge; black: the 6 other repetitions meeting the five selection criteria; grey: other 444 repetitions; vertical line: date of fences installation; horizontal dashed line: surface of the fenced area.

Despite a very large maximal geographical extent of the epidemics, the cases were highly aggregated in the selected repetition, both for pig herds and wild boar (Fig. 4). The local probability of infection was very high within the fenced area and on its western limit (where the primary case was introduced). It was high south of the fenced area, and low but not nil north and east of that area. It was nil everywhere else, except where a few pig herds has been infected in some of the stochastic model repetitions. 

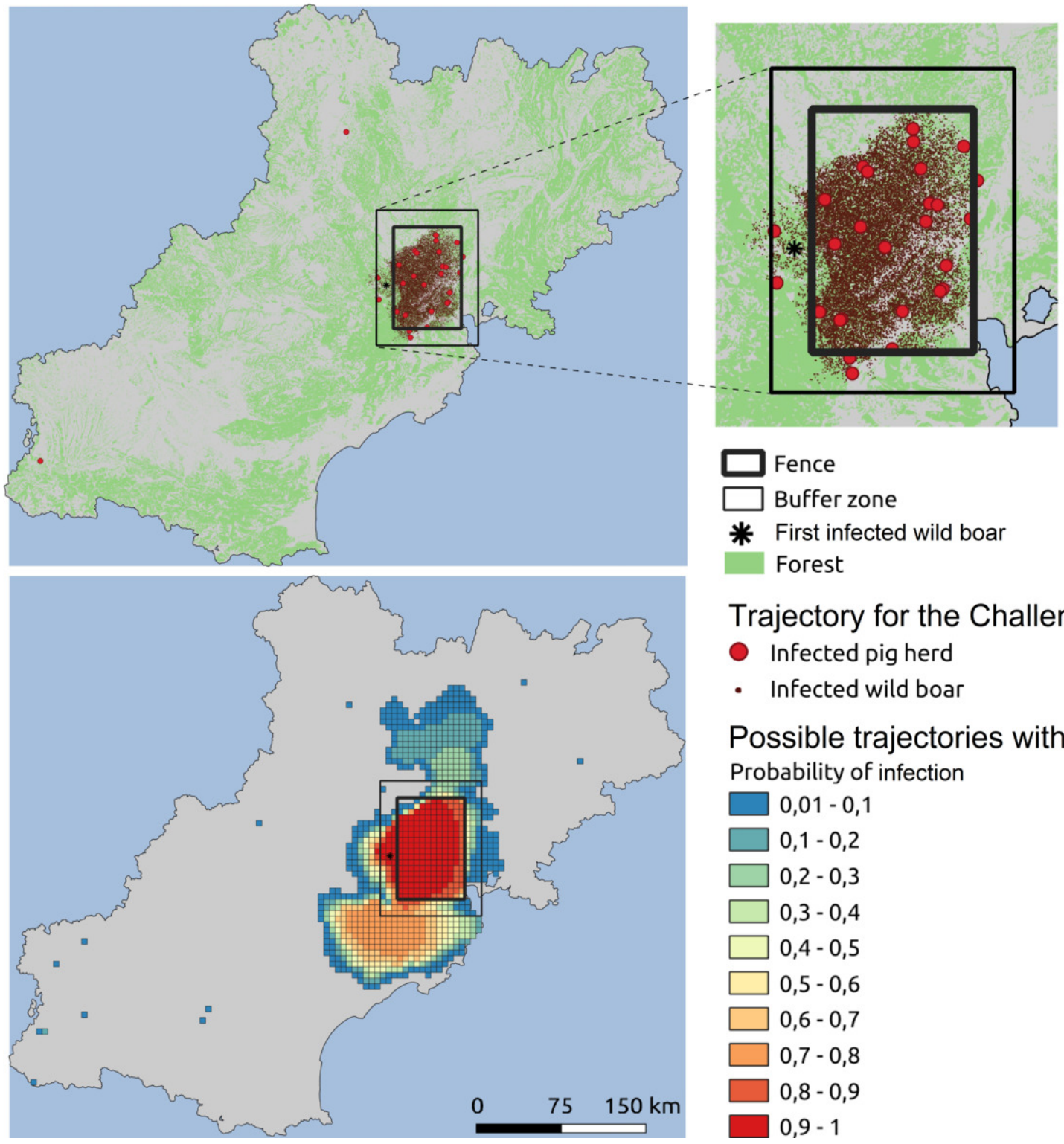

\section{Trajectory for the Challenge \\ - Infected pig herd \\ - Infected wild boar}

\section{Possible trajectories with M0}

Probability of infection

$\square$ 0,01-0,1

$\square 0,1-0,2$

$\square \quad 0,2-0,3$

$\square 0,3-0,4$

$\square$ 0,4-0,5

$\square$ 0,5-0,6

$\square \quad 0,6-0,7$

$\square \quad 0,7-0,8$

$\square 0,8-0,9$

0,9-1

Figure 4. Spatial distribution of infected epidemiological units. Top: cumulated infected epidemiological units 230 days after the first detection in the selected repetition (in red: pig herds, in brown: wild boar); Bottom: local probability of infection calculated on the 451 stochastic repetitions of the model, at a $5 \times 5 \mathrm{~km}^{2}$ scale.

\subsection{Data for players}

Before the ASF-Challenge started, the ASF-Challenge teams were provided with the available population data, i.e., the hunting bag size per department, as well as the farm database that comprised the location and characteristics of the pig farms (except for their biosecurity score). To allow the teams to become familiar with the data format and start developing their analytical pipelines before the actual start of the ASF-Challenge, dummy datasets were released to the participants on the movement data of live pigs between farms and on ASF surveillance outputs for a period of four weeks. 
The challenge was organized in three different epidemiological phases, phase 1, 2 and 3, which started when 50, 80 and 110 days had passed since the first detection, respectively. At the beginning of each phase, the ASF-Challenge teams were provided with the surveillance outputs and a situation report.

\subsubsection{Surveillance outputs}

The epidemiological information that was released to the participants comprised two datasets in CSV format: the list of cases (detected pig farms and wild boar) and the list of hunted wild boar that tested negative. Both datasets had the same structure. They included the ID of the herd (NA if a wild boar), the host type (pig farm or wild boar), the geographical coordinates, the detection method, the date of suspicion, the date of confirmation and the date of culling (NA if a wild boar). Note that the ID of the herd was the key to retrieve a farm's characteristics from

374 the farm database.

375 Overall, the synthetic data provided to the ASF-Challenge teams relied on a few detected cases in pig herds, spread over the challenge period, and a massive observed epidemic in wild boar. The number of detected cases highly correlated with the number of actual cases (Fig. 2), but only represented a small fraction of the epidemic, especially in wild boar (Fig. 5). After the implementation of fencing and increased hunting in the fenced area, the number of detected wild boar cases was much greater. The main cause of detection was active search before the implementation of fencing, tests on hunted animals after.

The full datasets related to hunting bags, pig farms, live movements and ASF surveillance outputs for the three phases are available as supplementary material (see link to public repository in SI1). 


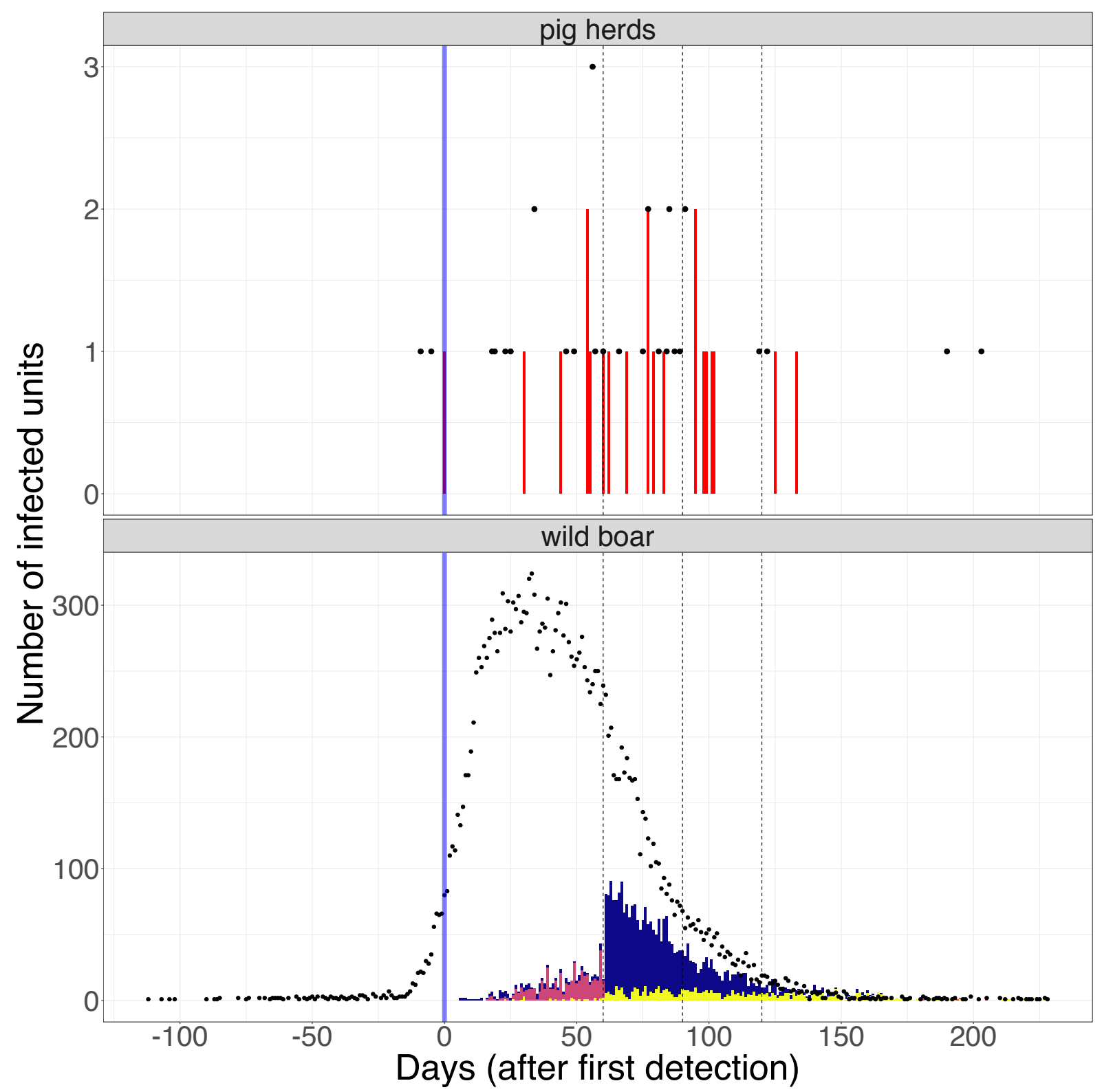

Figure 5. Total (dots) vs. detected (bars) number of cases in pig herds (top) and wild boar (bottom) over time in the synthetic data provided to the ASF-Challenge teams. The blue vertical line represents the date of the first detection. The cause of detection is shown for wild boar: tests on hunted wild boar (blue) and infected carcasses found either through passive surveillance (yellow) or through active search (red). See supplementary information (SI8 and Movie S1) for a dynamic view of the synthetic epidemic.

\subsubsection{Situation reports}

With each of the three data releases, narrative situation reports were provided to the teams. These reports aimed to contextualize the development of the epidemic during the period that just passed and summarize the different control alternatives. They usually included:

- a description of the interventions put in place since the beginning of the period;

- a narrative and qualitative summary of the epidemiological situation since the beginning of the period;

- a description of what was expected from the modelling teams (see below). 
A link to the three situation reports, released on days 50, 80 and 110 of the observed epidemic, is included in the supplementary information (SI7).

\subsubsection{Participant missions}

At the end of phase 1, i.e., 50 days after the first detection, participants were asked to 1) predict the number and location of wild boar cases and outbreaks in farms that should be expected during the following four weeks; 2) predict the effectiveness of fencing the infected zone (the precise location of the fence was provided to the teams), and 3) advise on whether hunting pressure should be increased in the fenced area.

At the end of phase 2, i.e., 80 days after the first detection, participants were asked to 1) update their predictions for the effectiveness of the fences implemented with or without the increased hunting pressure, now including a buffer zone of $15 \mathrm{~km}$ outside the fences, and 2) predict the effectiveness of five alternative control options, which were as follows:

- Culling of all pig herds located within a $3 \mathrm{~km}$ radius of infected farms;

- Increasing the size of the active search area around infected wild boar carcasses found outside the fenced/buffer areas (from $1 \mathrm{~km}$ to $2 \mathrm{~km}$ );

- Culling of all pig herds located within a $3 \mathrm{~km}$ radius of positive wild boar carcasses;

- Increasing the size of the surveillance zone (from $10 \mathrm{~km}$ to $15 \mathrm{~km}$, but maintaining the surveillance period of 30 days);

- Culling of all herds that traded pigs with an infected farm less than three weeks before detection.

Finally, at the end of phase 3, i.e., 110 days after the first detection, participants were asked to 1) update their predictions for the effectiveness of the fences implemented with or without the increased hunting pressure, 2) estimate the likelihood that the epidemic would fade out in the coming four months given the new control measures implemented, and 3) flag any long-term risk (i.e., second wave, risk of endemicity in wild boar, etc.) and advise on how to mitigate this risk.

At the end of each phase, participants had four weeks to provide their model outputs and address the questions.

\subsection{Expected predictions}

As measures implemented varied across periods of the ASF-Challenge, data provided for period $\mathrm{X}+1$ cannot be used to assess predictions from the teams based on data in period X. Fig. 6 shows the synthetic data provided to the ASF-Challenge teams together with the continuation of the prediction if nothing was changed in the next period. This highlighted the huge impact of fences and increased hunting (Fig. 6: period 1). In contrast, the alternative measures tested with the model had a very low impact. While we kept the most effective one (preventive culling of pig herds within $3 \mathrm{~km}$ of any positive wild boar), the predictions with and without this complementary measure were very similar (Fig. 6: period 2). 


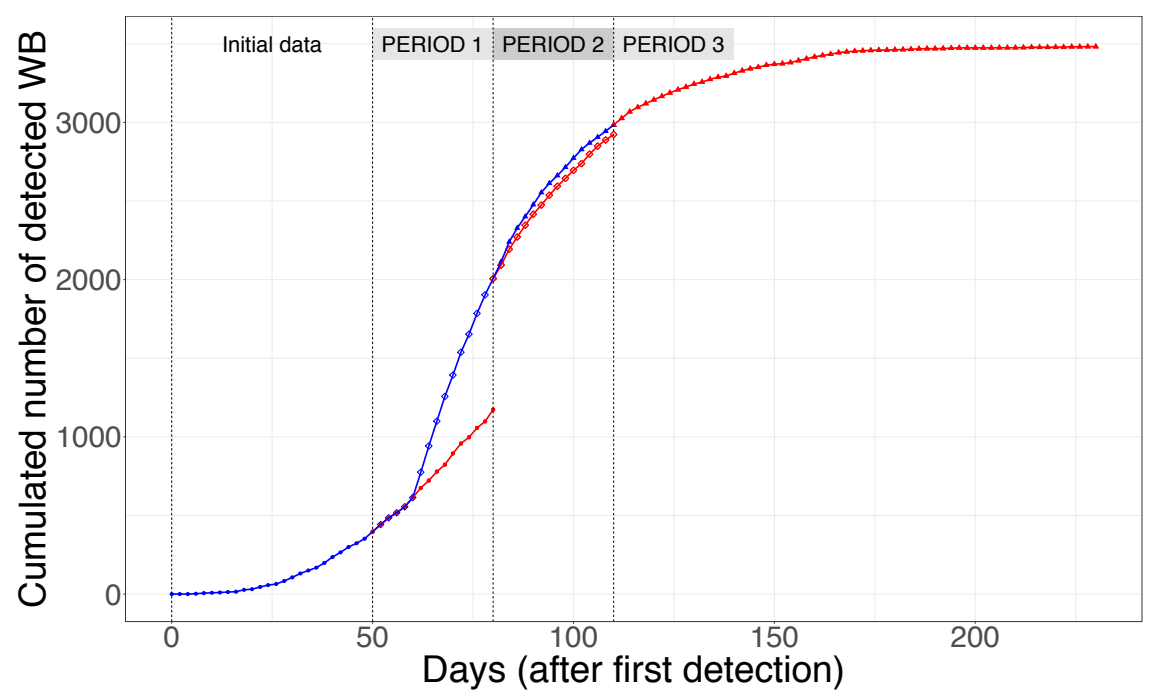

Figure 6. Synthetic data for the cumulative number of detected wild boar as provided to the ASF-Challenge teams (blue) and expected predictions with identical control conditions as the previous period (red). Vertical dotted lines show the period limits.

The very low number of infected wild boar detected 230 days after the first detection (Fig. 5) could be seen as an indication of a highly probable epidemic fade-out. However, the distribution of the number of infected carcasses of wild boar (Fig. 7) at this same date clearly showed that the probability of extinction was indeed very low.

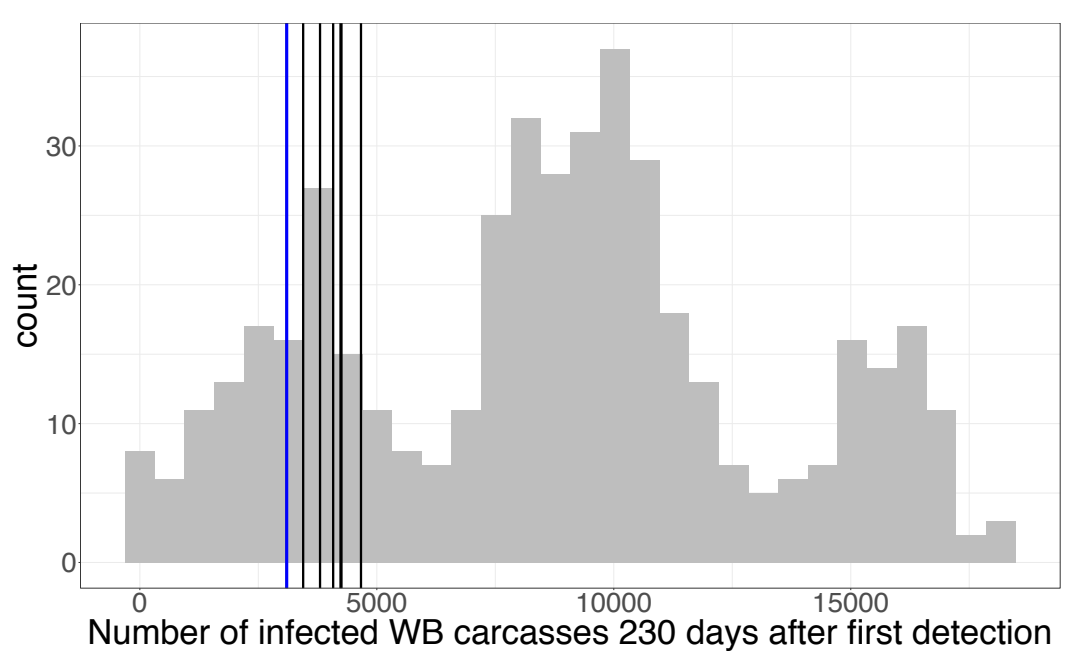

Figure 7. Distribution of the number of infected wild boar carcasses on the island 230 days after the first detection over the 451 stochastic model repetitions. Vertical lines: in blue, repetition selected for the ASF-Challenge; in black, the 6 other repetitions meeting the five selection criteria.

\section{Discussion}

Several modelling challenges have been organized over the past 10 years on infectious diseases of public health concern, such as Ebola, influenza, dengue and chikungunya. They have been described as a unique framework allowing the development and evaluation of modelling approaches and forecasting methodologies that would not be possible in the context of 
retrospective analyses of historical epidemics (Ajelli et al. 2018). In the context of infectious livestock diseases, no modelling challenges previously have been organized. This gap is particularly problematic since livestock populations and their pathogens have particular features that likely require specific modelling approaches and preparedness (Brooks-Pollock et al., 2015; Ezanno et al., 2020). This ASF modelling challenge was meant to fill this gap by allowing modelling teams to organize, get to know each other, develop new forecasting methodologies that could be deployed to investigate ASF epidemics, and compare their modelling approaches to that of the other teams. In this discussion, we synthesize the lessons that we learnt through organizing this first modelling challenge in animal health.

For this first challenge, we focused on an ASF epidemic due to the global risk posed by this disease to the pork industry and the important role played by the wildlife reservoir in the epidemiology of the virus (Dixon et al., 2020). However, we did not aim to reproduce a "true" ASF epidemic since there seems to be almost as many ASF epidemic patterns as there are contextual situations (Sauter-Louis et al., 2021). We simply aimed to generate an epidemic that "looked like" an ASF epidemic by considering the right populations (domestic pigs and wild boar) and appropriate transmission processes within and between the two populations. We acknowledge that the calibrated model that was used to generate the synthetic data is only one of a virtually infinite set of models that could have generated ASF-like epidemic trajectories. We note that in our simulated environment, the virus was detected much more frequently in wild boar than in domestic pig farms, which is consistent with several observed epidemiological situations such as in the Baltic States, Poland and the Republic of Korea (Sauter-Louis et al., 2021). Yet while it is important that the contextual situation represents a credible scenario inspired by real situations, there is no need to aim for a "perfectly calibrated" epidemic that likely does not exist.

To make the modelling challenge interesting and useful, the model that generated the synthetic data necessarily needed to be more detailed than the different models that were to be used to reproduce the data and make predictions. Since the challenge teams had not yet been recruited when the model was developed, we had no preconceived idea of the modelling approaches the different teams would use. To generate the synthetic data, we therefore developed a highly complex stochastic individual-based transmission model with 93 parameters and seven different transmission processes that very likely would be totally irrelevant for estimating transmission parameters and making useful predictions. The consequences of this complexity were that 1) the data generation and database preparation were considerably computationally intensive, and 2) it was extremely time-consuming to calibrate the model in order to generate "ASF-like" epidemic trajectories that met the pre-defined selection criteria. In particular, the lack of data with regards to wild boar population dynamics and mobility patterns leaves no room for contrasting assumptions, and makes it difficult to anticipate how to account for an impact of fences on virus spread. The interface between livestock and wildlife, while crucial for better understanding multi-host diseases, is still poorly observed (Vincente et al. 2021).

Using synthetic data for a modelling challenge also renders it possible to control the "fog of war", i.e., to know precisely the extent to which the detection data provided to modelling teams mirrors the infection data in both host populations. Here, we did not attempt to generate several scenarios for this "fog of war", as done in the Ebola forecasting challenge (Viboud et al., 2018), since the objective was not to assess how fog of war could impact model predictions. We decided to create only one scenario that aimed to introduce sufficient uncertainty to mimic a realistic situation. A few pig herds were not initially in the farm database. Detection was far from perfect in both host populations, especially at the start of the epidemic and in wild boar. 
Similar to the RAPIDD Ebola forecasting challenge (Ajelli et al., 2018), we documented the data format by preparing "Read Me" documents describing the structure of all of the different datasets and the interpretation of all of the different variables within each dataset (available in the code repository, see supplementary information SI1). A dummy set of data and this "Read Me" document were sent to the different modelling teams four weeks before the start of the challenge so that the teams could become familiar with the data format and start developing their analytical pipelines. We realized that this step was extremely important to motivate the teams and optimize their readiness.

\section{Declaration of interest:}

The authors have no conflict of interest.

\section{Funding}

This work was supported by the animal health division of INRAE [PPApred grant].

\section{Author contribution}

SP: Conceptualization; Formal analysis; Investigation; Methodology; Software; Supervision; Validation; Visualization; Writing the original draft

TV: Conceptualization; Data curation; Formal analysis; Investigation; Methodology; Supervision; Validation; Visualization; Writing the original draft

MM: Data curation; Formal analysis; Methodology; Investigation; Visualization; Writing review \& editing

\section{SB: Formal analysis; Investigation; Methodology; Visualization; Writing - review \& editing}

PE: Conceptualization; Formal analysis; Funding acquisition; Investigation; Methodology; Project administration; Resources; Software; Supervision; Validation; Visualization; Writing the original draft

\section{Acknowledgements}

C. Belloc \& C. Peroz (Oniris), E. Gilot-Fromont (VetAgroSup), E. Baubet \& E. Marboutin (OFB) are all thanked for their expertise on ASF, pig herd management, and wild boar population dynamics, which greatly helped us to define the model and associated scenarios.

We are most grateful to the Bioinformatics Core Facility of Nantes BiRD, member of Biogenouest, Institut Français de Bioinformatique (IFB) (ANR-11-INBS-0013), and to the INRAE MIGALE bioinformatics facility (MIGALE, INRAE, 2020. Migale bioinformatics Facility, doi: 10.15454/1.5572390655343293E12), for the use of their computing resources.

\section{References}

Ajelli M, et al. (2018) The RAPIDD Ebola forecasting challenge: Model description and synthetic data generation. Epidemics 22:3-12. https://doi.org/10.1016/j.epidem.2017.09.001 Brooks-Pollock, E., de Jong, M.C.M., Keeling, M.J., Klinkenberg, D., Wood, J.L.N., 2015. Eight challenges in modelling infectious livestock diseases. Epidemics 10, 1-5. https://doi.org/10.1016/j.epidem.2014.08.005 
Chretien, J.-P., George, D., Shaman, J., Chitale, R.A., McKenzie, F.E., 2014. Influenza forecasting in human populations: a scoping review. Plos One 9:e94130. https://oi.org/10.1371/journal.pone.0094130.

Chretien, J.-P., Riley, S., George, D.B., 2015. Mathematical modeling of the West Africa ebola epidemic. ELife 4:e09186. https://doi.org/10.7554/eLife.09186.

Coker, T.L.R., Rozsypálek, J., Edwards, A., Harwood, T.P., Butfoy, L., Buggs, R.J.A., 2019. Estimating mortality rates of European ash (Fraxinus excelsior) under the ash dieback (Hymenoscyphus fraxineus) epidemic. Plants, People, Planet 1, 48-58. https://doi.org/10.1002/ppp3.11.

Del Valle, S.Y., McMahon, B.H., Asher, J., Hatchett, R., Lega, J.C., Brown, H.E., Leany, M.E., Pantazis, Y., Roberts, D.J., Moore, S., Peterson, A.T., Escobar, L.E., Qiao, H., Hengartner, N.W., Mukundan, H., 2018. Summary results of the 2014-2015 DARPA Chikungunya challenge. BMC Infect. Dis. 18, 245. https://doi.org/10.1186/s12879-018-3124-7.

Dixon, L.K., Stahl, K., Jori, F., Vial, L., Pfeiffer, D.U., 2020. African Swine Fever Epidemiology and Control. Annual Review of Animal Biosciences, 8, 221-246. https://doi.org/10.1146/annurev-animal-021419-083741.

EFSA Panel on Animal Health and Welfare (AHAW), Nielsen, S.S., Alvarez, J., Bicout, D.J., Calistri, P., Canali, E., Drewe, J.A., Garin-Bastuji, B., Gonzales Rojas, J.L., Schmidt, C., Herskin, M., Michel, V., Padalino, B., Pasquali, P., Roberts, H.C., Spoolder, H., Stahl, K., Velarde, A., Winckler, C., Blome, S., Boklund, A., Bøtner, A., Dhollander, S., Rapagnà, C., Van der Stede, Y., Miranda Chueca, M.A., 2021. Research priorities to fill knowledge gaps in wild boar management measures that could improve the control of African swine fever in wild boar populations. EFSA J. 19(7):e06716. https://doi.org/10.2903/j.efsa.2021.6716.

European Food Safety Authority (EFSA), Boklund, A., Bøtner, A., Chesnoiu, V.T., Depner, K., Desmecht, D., Guberti, V., Helyes, G., Korytarova, D., Linden, A., Miteva, A., More, S., Olsevskis, E., Ostojic, S., Roberts, H., Spiridon, M., Stahl, K., Thulke, H.-H., Vilija, G., Viltrop, A., Wallo, R., Wozniakowski, G., Abrahantes Cortinas, J., Dhollander, S., Gogin, A., Ivanciu, C., Papanikolaou, A., Villeta Gonzalez, L.C., Gortazar Schmidt, C., 2020. Scientific report on the epidemiological analyses of African swine fever in the European Union (November 2018 to October 2019). EFSA J. 18(1):5996, 107 pp. https://doi.org/10.2903/j.efsa.2021.5996.

Ezanno, P., Andraud, M., Beaunée, G., Hoch, T., Krebs, S., Rault, A., Touzeau, S., Vergu, E., Widgren, S., 2020. How mechanistic modelling supports decision making for the control of enzootic infectious diseases. https://doi.org/10.1016/j.epidem.2020.100398.

Ezanno, P., Picault, S., Mancini, M., Bareille, S., Vergne, T. The ASF modelling challenge: model comparison and lessons learnt, submitted to Epidemics special issue on ASF modelling challenge.

Friedberg, I., Wass, M.N., Mooney, S.D., Radivojac, P., 2015. Ten simple rules for a community computational challenge. PLoS Comput. Biol. 11, 3-7. https://doi.org/10.1371/journal.pcbi.1004150.

Gortázar, C., Ferroglio, E., Höfle, U., Frölich, K., Vicente, J., 2007. Diseases shared between wildlife and livestock: a European perspective. Eur J Wildl Res 53, 241. https://doi.org/10.1007/s10344-007-0098-y.

Grassly, N.C., Fraser, C., 2008. Mathematical models of infectious disease transmission. Nat. Rev. Microbiol. 6, 477-487. https://doi.org/10.1038/nrmicro1845.

Halasa, T., Bøtner, A., Mortensen, S., Christensen, H., Toft, N., Boklund, A., 2016. Simulating the epidemiological and economic effects of an African swine fever epidemic in 
industrialized swine populations. Vet. Microbiol. 193, 7-16. https://doi.org/10.1016/j.vetmic.2016.08.004.

Hamer, W.H., 1906. Epidemic disease in England. Lancet I, 733-739.

Hayes, B.H., Andraud, M., Salazar, L.G., Rose, N., Vergne, T., 2021. Mechanistic modelling of African swine fever: A systematic review. Prev. Vet. Med. 191:105358. https://doi.org/10.1016/j.prevetmed.2021.105358.

Holmdahl, I., Buckee, C., 2020. Wrong but useful - what Covid-19 epidemiologic models can and cannot tell us. N. Engl. J. Med. 383:303-305. https://doi.org/10.1056/NEJMp2016822.

Johansson, M.A., Apfeldorf, K.M., Dobson, S., Devita, J., Buczak, A.L., Baugher, B., Moniz, L.J., Bagley, T., Babin, S.M., Guven, E., Yamana, T.K., Shaman, J., Moschou, T., Lothian, N., Lane, A., Osborne, G., Jiang, G., Brooks, L.C., Farrow, D.C., Hyun, S., Tibshirani, R.J., Rosenfeld, R., Lessler, J., Reich, N.G., Cummings, D.A.T., Lauer, S.A., Moore, S.M., Clapham, H.E., Lowe, R., Bailey, T.C., García-Díez, M., Carvalho, M.S., Rodó, X., Sardar, T., Paul, R., Ray, E.L., Sakrejda, K., Brown, A.C., Meng, X., Osoba, O., Vardavas, R., Manheim, D., Moore, M., Rao, D.M., Porco, T.C., Ackley, S., Liu, F., Worden, L., Convertino, M., Liu, Y., Reddy, A., Ortiz, E., Rivero, J., Brito, H., Juarrero, A., Johnson, L.R., Gramacy, R.B., Cohen, J.M., Mordecai, E.A., Murdock, C.C., Rohr, J.R., Ryan, S.J., Stewart-Ibarra, A.M., Weikel, D.P., Jutla, A., Khan, R., Poultney, M., Colwell, R.R., RiveraGarcía, B., Barker, C.M., Bell, J.E., Biggerstaff, M., Swerdlow, D., Mier-y-Teran-Romero, L., Forshey, B.M., Trtanj, J., Asher, J., Clay, M., Margolis, H.S., Hebbeler, A.M., George, D., Chretien, J.P., 2019. An open challenge to advance probabilistic forecasting for dengue epidemics. Proc. Nat. Acad. Sci. USA 116, 24268-24274. https://doi.org/10.1073/pnas.1909865116.

Jori, F., Massei, G., Licoppe, A., Ruiz-Fons, F., Linden, A., Václavek, P., Chenais, E., Rosell, C. 2021. Management of wild boar populations in the European Union before and during the ASF crisis. In: Understanding and combatting African Swine Fever: a European perspective, Iacolina, L., Penrith, M.-L., Bellini, S., Chenais, E., Jori, F., Montoya, M., Ståhl, K., GavierWidén, D. (Eds). pp. 197-228. https://doi.org/10.3920/978-90-8686-910-7_8.

Keeling, M.J., 2005. Models of foot-and-mouth disease. Proc. Roy. Soc. B 27̄2(1569), 11951202. https://doi.org/10.1098/rspb.2004.3046.

Lange M., 2015. Alternative control strategies against ASF in wild boar populations. EFSA supporting publication 2015:EN 843. 29 pp. https://doi.org/10.2903/sp.efsa.2015.EN-843.

Metcalf, C.J.E., Edmunds, W.J., Lessler, J., 2015. Six challenges in modelling for public health policy. Epidemics 10, 93-96. https://doi.org/10.1016/j.epidem.2014.08.008.

Nsoesie, E.O., Brownstein, J.S., Ramakrishnan, N., Marathe, M.V., 2014. A systematic review of studies on forecasting the dynamics of influenza outbreaks. Influenza and Other Respiratory Viruses 8, 309-316. https://doi.org/10.1111/irv.12226.

Parham, P.E., Waldock, J., Christophides, G.K., Hemming, D., Agusto, F., Evans, K.J., Fefferman, N., Gaff, H., Gumel, A., LaDeau, S., Lenhart, S., Mickens, R.E., Naumova, E.N., Ostfeld, R.S., Ready, P.D., Thomas, M.B., Velasco-Hernandez, J., Michael, E., 2015. Climate, environmental and socio-economic change: weighing up the balance in vectorborne disease transmission. Phil. Trans. R. Soc. B 370:20130551. https://doi.org/10.1098/rstb.2013.0551.

Picault, S., Huang, Y.-L., Sicard, V., Arnoux, S., Beaunée, G., Ezanno, P., 2019. EMULSION: Transparent and flexible multiscale stochastic models in human, animal and plant

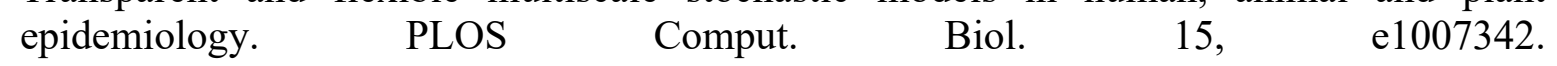
https://doi.org/10.1371/journal.pcbi.1007342

Reich, N.G., Brooksb, L.C., Foxc, S.J., Kandulad, S., McGowane, C.J., Moorea, E., Osthusf, D., Rayg, E.L., Tushara, A., Yamanad, T.K., Biggerstaffe, M., Johanssonh, M.A., 
Rosenfeldi, R., Shamand, J., 2019. A collaborative multiyear, multimodel assessment of seasonal influenza forecasting in the United States. Proc Natl Acad Sci USA 116:31463154. https://doi.org/10.1073/pnas.1812594116.

Rosenfeld, R., Grefenstette, J., Burke, D., 2013. A proposal for standardized evaluation of epidemiological models. http://delphi.midas.cs.cmu.edu/files/StandardizedEvaluation_Revised 12-11-09.pdf.

Rowlands, R. J., Michaud, V., Heath, L., Hutchings, G., Oura, C., Vosloo, W., Dwarka, R., Onashvili, T., Albina, E., Dixon, L. K., 2008. African Swine Fever Virus Isolate, Georgia, 2007. Emerg. Infect. Dis., 14(12), 1870-1874. https://doi.org/10.3201/eid1412.080591.

Saker, L., Kelley, L., Cannito, B., Gilmore, A., Campbell-Lendrum, D., 2004. Globalization and infectious diseases: a review of the linkages. World Health Organization Tech. rep. $\mathrm{TDR} / \mathrm{STR} / \mathrm{SEB} / \mathrm{ST} / 04.2$.

Sánchez-Cordón, P.J., Nunez, A., Neimanis, A., Wikström Lassa, E., Montoya, M., Crooke, H., Gavier Widén, D., 2019. African swine fever: Disease dynamics in wild boar experimentally infected with ASFV isolates belonging to genotype I and II. Viruses 11(9):852. https://doi.org/10.3390/v11090852.

Sauter-Louis, C., Conraths, F.J., Probst, C., Blohm, U., Schulz, K., Sehl, J., Fischer, M., Forth, J.H., Zani, L., Depner, K., Mettenleiter, T.C., Beer, M., Blome, S., 2021. African swine fever in wild boar in Europe - A Review. Viruses 13:1717. https://doi.org/10.3390/v13091717

Van Kerkhove, M.D., Ferguson, N.M., 2012. Epidemic and intervention modelling - a scientific rationale for policy decisions? Lessons from the 2009 influenza pandemic. Bull. World Health Organ. 90, 306-310. https://doi.org/10.2471/BLT.11.097949.

Vergne, T., Chen-Fu, C., Li, S., Cappelle, J., Edwards, J., Martin, V., Pfeiffer, D.U., Fusheng, G., Roger, F.L., 2017. Pig empire under infectious threat: risk of African swine fever introduction into the People's Republic of China. Vet. Rec. 181(5):117. https://doi.org/10.1136/vr.103950.

Vetter, S.G., Puskas, Z., Bieber, C., Ruf, T., 2020. How climate change and wildlife management affect population structure in wild boars. Sci. Rep. 10, 7298. https://doi.org/10.1038/s41598-020-64216-9.

Viboud, C., Sun, K., Gaffey, R., Ajelli, M., Fumanelli, L., Merler, S., Zhang, Q., Chowell, G., Simonsen, L., Vespignani, A., 2018. The RAPIDD ebola forecasting challenge: Synthesis and lessons learnt. Epidemics 22, 13-21. http://dx.doi.org/10.1016/j.epidem.2017.08.002.

Viboud, C., Vespignani, A., 2019. The future of influenza forecasts. Proc Natl Acad Sci USA 116(8):2802-2804. https://doi.org/10.1073/pnas.1822167116.

Vincente, J., Vercauteren, K.C., Gortazar C., 2021. Diseases at the Wildlife - Livestock Interface: Research and Perspectives in a Changing World. Springer Cham, Wildlife Research Monographs, 421p. https://doi.org/10.1007/978-3-030-65365-1 